\title{
Simultaneous activation of multiple vestibular pathways upon electrical stimulation of semicircular canal afferents
}

\author{
Anissa Boutabla ${ }^{1}\left[\right.$ - Samuel Cavuscens ${ }^{1} \cdot$ Maurizio Ranieri ${ }^{1} \cdot$ Céline Crétallaz $^{1} \cdot$ Herman Kingma $^{2,3}$ (1)

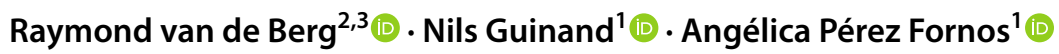

Received: 16 April 2020 / Revised: 2 July 2020 / Accepted: 27 July 2020 / Published online: 10 August 2020

(c) The Author(s) 2020

\begin{abstract}
Background and purpose Vestibular implants seem to be a promising treatment for patients suffering from severe bilateral vestibulopathy. To optimize outcomes, we need to investigate how, and to which extent, the different vestibular pathways are activated. Here we characterized the simultaneous responses to electrical stimuli of three different vestibular pathways. Methods Three vestibular implant recipients were included. First, activation thresholds and amplitude growth functions of electrically evoked vestibulo-ocular reflexes (eVOR), cervical myogenic potentials (ecVEMPs) and vestibular percepts (vestibulo-thalamo-cortical, VTC) were recorded upon stimulation with single, biphasic current pulses (200 $\mu$ s/phase) delivered through five different vestibular electrodes. Latencies of eVOR and ecVEMPs were also characterized. Then we compared the amplitude growth functions of the three pathways using different stimulation profiles (1-pulse, $200 \mu$ s/phase; 1-pulse, $50 \mu \mathrm{s} /$ phase; 4-pulses, $50 \mu \mathrm{s} /$ phase, 1600 pulses-per-second) in one patient (two electrodes).

Results The median latencies of the eVOR and ecVEMPs were $8 \mathrm{~ms}$ (8-9 ms) and 10.2 ms (9.6-11.8 ms), respectively. While the amplitude of eVOR and ecVEMP responses increased with increasing stimulation current, the VTC pathway showed a different, step-like behavior. In this study, the $200 \mu \mathrm{s} /$ phase paradigm appeared to give the best balance to enhance responses at lower stimulation currents.

Conclusions This study is a first attempt to evaluate the simultaneous activation of different vestibular pathways. However, this issue deserves further and more detailed investigation to determine the actual possibility of selective stimulation of a given pathway, as well as the functional impact of the contribution of each pathway to the overall rehabilitation process.
\end{abstract}

Keywords Bilateral vestibulopathy · Vestibular implant · Vestibulo-ocular reflex · Vestibulo-spinal reflex ·

Neuroprosthesis · Electrical stimulation

\section{Introduction}

Bilateral vestibulopathy, a bilaterally reduced or absent vestibular function, causes disabling symptoms such as imbalance and oscillopsia. It has a direct impact on the

Angélica Pérez Fornos

angelica.perez-fornos@hcuge.ch

1 Division of Otorhinolaryngology Head and Neck Surgery, Geneva University Hospitals and University of Geneva, Geneva, Switzerland

2 Division of Balance Disorders, Department of ENT, Maastricht University Medical Centre, Maastricht, The Netherlands

3 Faculty of Physics, Tomsk State Research University, Tomsk, Russia quality of life in at least three million people worldwide $[1,2]$. Currently, this pathology remains poorly understood and treatment options are limited [3,4]. Our group attempts to develop a treatment alternative for these patients: a vestibular implant. The concept is to use electrical currents to transmit head motion information normally detected by the vestibular system, to the vestibular nerve $[5,6]$. This is comparable to the concept of the cochlear implant for hearing rehabilitation. Briefly, the vestibular implant comprises an implanted neural stimulator incorporating electrodes to be positioned close to the branches of the vestibular nerve [5]. The stimulator applies electrical currents through these vestibular electrodes that can be modulated in intensity or rate using motion sensors attached to the patient's head. The electrical activity delivered by the device thus signals the 
speed and direction of head movements, replacing the damaged vestibular system [7].

The feasibility of this concept was first established in several animal studies, starting in the 1960s [8, 9]. More recent feasibility studies using animal models demonstrated the effectiveness of using chronically implantable devices to generate controlled vestibulo-ocular responses [10-13]. Special stimulation paradigms were developed to improve outcomes (e.g., peak eye velocities, alignment) [10-13]. Other studies investigated fundamental aspects of vestibular function such as adaptation and plasticity $[14,15]$, as well as how the different vestibular reflexes complement each other to achieve complex behavior (e.g., contribution of the vestibulo-ocular and vestibulo-spinal reflexes to gaze stabilization) [16]. Since then, other approaches have been developed to expand the original approach targeting semicircular canals exclusively to also attempt stimulation of the otolith organs [17]. Human research also made important progress in recent years [18, 19]. Special implantable stimulators were developed and were progressively improved, together with the surgical approach for optimal and safe implantation of the device [20-23]. The most recent results on human patients demonstrated that it is possible to restore vestibular reflexes [24-28], to significantly improve visual abilities in dynamic conditions (i.e., while walking) [29] and to generate controlled postural responses in implanted patients [30,31]. Other studies demonstrated the feasibility of using long-term electrical stimulation to stimulate the vestibular pathways, both with systems designed for stimulation of the semicircular canals or the otolithic organs [17, 27, 32]. Taken together, these results pave the way for the clinical application of the vestibular implant.

The optimization of outcomes achieved with the vestibular implant requires that the multidimensional nature of the "balance system" is considered. After all, balance is an important "sixth sense" which substantially relies on the parallel activation of multiple vestibular pathways. These pathways start at the peripheral vestibular system and project to the vestibular nuclei in the brainstem. These nuclei are the neural relay of the vestibular pathways where information from other sensory systems (i.e., vision, proprioception) also converge. The central neurons of the vestibular nuclei project to different structures that generate reflexes for gaze stabilization (vestibulo-ocular reflex) [33] and for postural control during everyday activities (vestibulo-collic and vestibulo-spinal reflexes) [34-36]. Finally, vestibulothalamo-cortical (VTC) pathways carry information to the brain which combines vestibular and extra-vestibular cues to ensure more complex central functions, such as self-motion perception or spatial navigation. While previous studies have addressed each of these pathways individually, the purpose of this study was to simultaneously explore the relationships between the electrical stimulus delivered to the vestibular system and the resulting responses of the different vestibular pathways. Specifically, we attempted to investigate the main characteristics and the amplitude growth function (amplitude of the response as a function of stimulation current) of three vestibular pathways: vestibulo-ocular reflex (VOR), vestibulo-collic reflex (VCR), and vestibulo-thalamo-cortical (VTC) elicited with a set of controlled electrical stimuli. This study follows up on our previous investigations attempting to determine the most efficient stimulation paradigm to optimize outcomes with the vestibular implant [37]. Shorter phase durations $(<200 \mu$ s) and, to a lesser extent, slower pulse rates $(<200$ pulses-per-second, pps) allowed maximizing the electrical dynamic range available for eliciting a wider range of intensities of vestibular percepts. Interestingly, however, this observation was not consistent for VOR responses. In this case, the main factor allowing to maximize the response was modulation depth (i.e., amplitude of the modulation signal), while variations in phase duration and pulse rate appeared less effective than previously reported in animals $[11,13]$. The present study attempts to go one step further, and assess the variability of responses of different pathways elicited simultaneously using different stimulation profiles.

\section{Methods}

\section{Subjects, device and surgery}

Three bilateral vestibulopathy patients who previously received a vestibular implant prototype [7], participated in this study. Details on the inclusion criteria, device and surgical procedures can be found in previous publications $[5,7,24]$. Briefly, the device consisted of a modified cochlear implant (MED-EL, Innsbruck, Austria) providing one to three extra-cochlear electrodes for vestibular stimulation (Table 1). These vestibular electrodes were implanted in the vicinity of the lateral, posterior and superior ampullary branches of the vestibular nerve (respectively, LAN, PAN and SAN) using an intralabyrinthine or extralabyrinthine surgical approach $[20,23,38]$. Note that the PAN electrodes in patients $\mathrm{S} 1$ and $\mathrm{S} 2$ were not tested during the experiments presented here (grayed out in Table 1). Stimulation with these electrodes did not evoke any vestibular responses even at the highest current levels available for safe stimulation. This is probably due to the traumatic etiology of these cases (temporal bone fracture going through the ampulla of the PAN).

All patients were recruited at the Division of Otorhinolaryngology and Head and Neck Surgery of the Geneva University Hospitals. Note that only one vestibular electrode was activated at a time for a given experimental trial. All 
Table 1 Main demographic characteristics of the three patients participating in this study

\begin{tabular}{|c|c|c|c|c|c|c|c|c|}
\hline Patients & Sex & Etiology & Onset & $\begin{array}{l}\text { Age at } \\
\text { implanta- } \\
\text { tion }\end{array}$ & Year implanted & Implanted side & Vestibular electrodes & $\begin{array}{l}\text { Surgical } \\
\text { approach }\end{array}$ \\
\hline $\mathrm{S} 1$ & $\mathrm{~F}$ & Traumatic & Acute $(<1$ year $)$ & 67 & 2013 & Left & PAN/LAN/SAN & IL \\
\hline $\mathrm{S} 2$ & M & Traumatic & Acute ( 3 years $)$ & 53 & 2015 & Right & PAN/LAN/SAN & $\mathrm{IL}$ \\
\hline S3 & M & Congenital/idiopathic & Progressive & 46 & 2008 & Left & PAN & EL \\
\hline
\end{tabular}

$M$ male, $F$ female, $P A N$ posterior ampullary nerve, $L A N$ lateral ampullary nerve, $S A N$ superior ampullary nerve, $E L$ extralabyrinthic [20], $I L$ intralabyrinthic [21]

cochlear electrodes were switched off during the experimental procedures.

\section{Electrical stimulation}

The setup for the electrical stimulation was composed of a computer running custom software based on MATLAB R2014b (The Mathworks Inc., Natick, Massachusetts, USA). This software allowed customization of stimulation parameters (current intensity, pulse rate, phase width, electrode, current range, train pulse characterization, etc.). The computer communicated this information to the implanted stimulator via a special interface device (dRIB; MED-EL, Innsbruck, Austria) and the system's antenna.

Each experimental trial consisted of 100 electrical stimuli presented at a repetition rate of $5 \mathrm{~Hz}$. The electrical stimuli involved one or several cathodic-first, biphasic, charge balanced pulses delivered to the vestibular nerve with one of the implanted vestibular electrodes. First, we investigated the growth function of the responses of the three vestibular pathways (VOR, VCR, and VTC), using a single pulse with $200 \mu$ s phase duration (S1-SAN, S1-LAN, S2-SAN, S2-LAN and S3-PAN). Second, we compared the growth functions obtained with three different stimulation paradigms on one subject (S1-SAN and S1-LAN) who was available for this additional experiment. The three stimulation paradigms were (1) a single pulse with a phase of $200 \mu$ s (as used in the previous experiment and in our preceding studies [37]); (2) a single pulse with a short phase of $50 \mu$ s (similar to that commonly used in clinical cochlear implant fittings); and (3) a pulse train of four $50 \mu \mathrm{s} /$ phase pulses presented at rate of 1600 pulses-per-second (pps) (total charge per stimulation trial equal to the single pulse with a $200 \mu$ s phase duration).

\section{Characteristics and growth functions of the VOR, VSR, and VTC pathways}

First, a measurement without any electrical stimulation $(0 \mu \mathrm{A})$ was performed to record baseline response levels (e.g., noise). Then consecutive experimental trials were performed with increasing current amplitude (steps of $50 \mu \mathrm{A}$ ) to investigate the characteristics and the growth functions of each vestibular response, up to the upper comfortable level (UCL). The UCL is defined to be the current level immediately below the level where undesired effects are observed (i.e., facial nerve activation, uncomfortably loud sound) or at the maximum safe current level allowed by the device, similar to our previous studies [7]. Note that this experimental design involving very short stimulation trials did not comprise special psychophysical paradigms to compensate for adaptation effects (e.g., ascending/descending) which would have resulted in increased experimental times. In our experimental conditions, the bias induced by increased experimental time would have been greater than that induced by any potential adaptation effects.

Figure 1 shows an example of one of the recordings for $\mathrm{S} 1$ obtained upon electrical stimulation of the vestibular electrode implanted in the proximity of the SAN. The markers in the figure illustrate the different time points that were considered in the analysis of latency and amplitude of the different responses, explained in detail below.

Electrically evoked VOR responses (eVOR) were recorded using a binocular, video-based eye tracking system at a high sampling rate $(1000 \mathrm{~Hz})$ to allow acquisition of short-latency eye-movements (EyeLink 1000 Plus; SR Research, Ottawa, Canada). Some patients suffered from strabismus which hindered accurate binocular fixation; therefore, only the dominant eye was recorded in all subjects. Each experimental trial started with a calibration procedure which consisted of nine sequential fixations of a dot moving randomly around the computer screen borders, followed by a similar nine-point validation procedure to ensure calibration accuracy $\left(\right.$ error $\left.<0.1^{\circ}\right)$. Horizontal and vertical eye velocity data from the EyeLink system were imported to MATLAB R2018b. Peak eye velocity (PEV) of the signal was calculated as the square root of the sums of the squares of horizontal and vertical eye movements. Trials including artefacts (saccades or blinks) were manually removed. Then the average of artefact-free PEV responses was calculated. The latency of the electrically evoked vestibulo-ocular reflex (eVOR) is determined by the beginning of the eye movement, calculated as the first inflection point of the total PEV signal ( $\mathrm{LAT}_{\mathrm{T}} \mathrm{eVOR}$ ). The consecutive eVOR peaks were determined using the maximum or minimum, depending on 

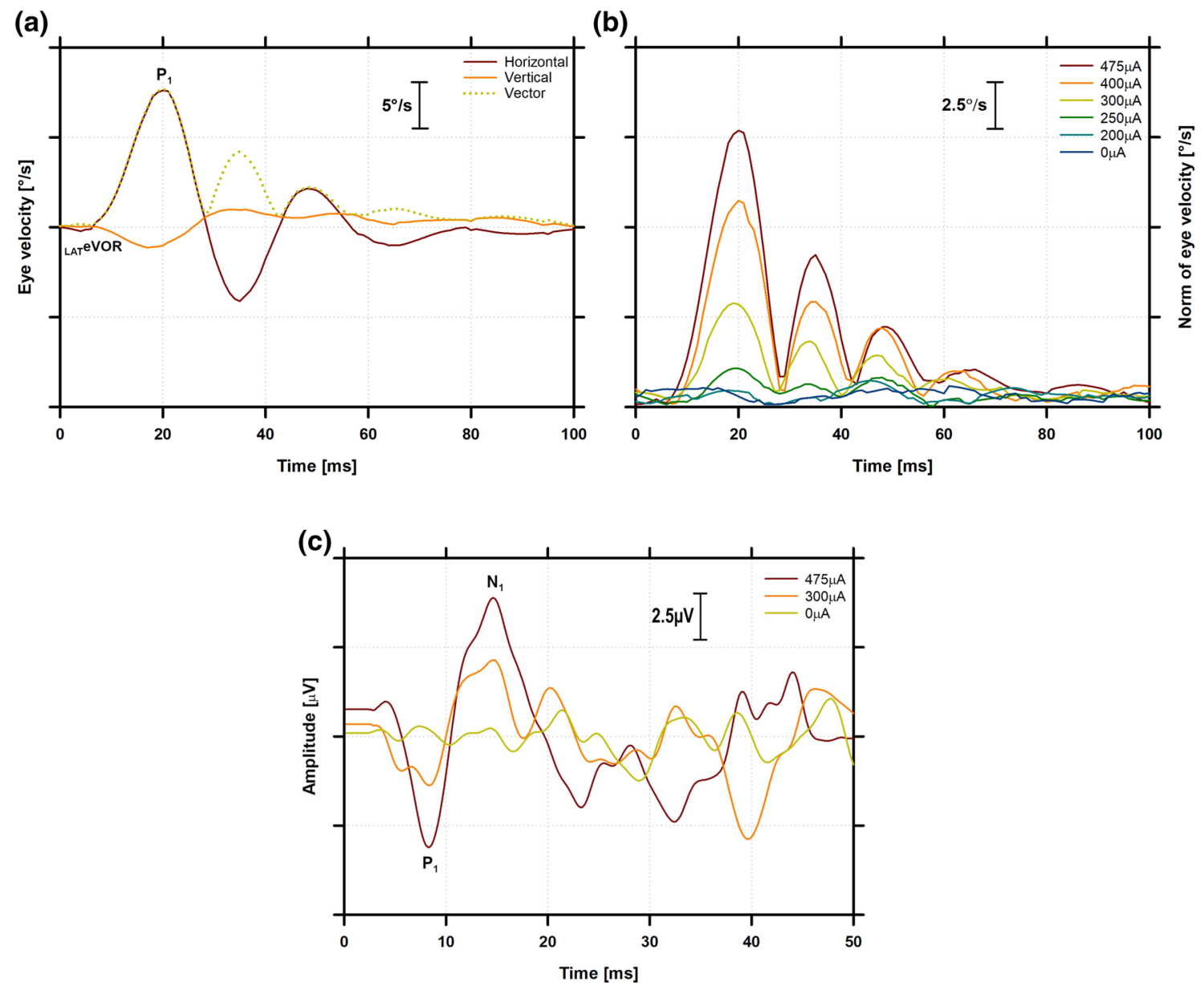

Fig. 1 Example of recordings and data processing of results obtained in patient S1 (SAN electrode) upon stimulation with a charge-balanced, cathodic-first, biphasic current pulse of $200 \mu$ s per phase. a Horizontal, vertical, and vector norm components of the averaged eVOR signal (respectively, solid dark red, solid orange, and dotted green lines). ${ }_{\mathrm{LAT}} \mathrm{eVOR}$ marks the beginning of the eye movement (i.e., the latency), and $P_{1}$ marks the first peak of the total peak eye velocity vector $\left(P_{1} \mathrm{VOR}\right)$. b Example of the evolution of the norm of

signal polarity, of the second derivate of the total PEV signal (see Fig. 1a).

Electrical cervical vestibular evoked myogenic potentials (ecVEMPs) were recorded with the NeuroAudio system (Neurosoft, Ivanovo, Russian Federation), with the active recording electrodes positioned on the main belly of the sternocleidomastoid muscle (SCM), approximately equidistant from the mastoid process and the sternum. The ground electrode was placed on the superior part of the sternum, and the indifferent electrode on the forehead. Instead of being in the standard supine position and lifting the head, we had patients sit down with the head placed on a head support tower. The patient was requested to look straight ahead to a 24 " computer screen (XL2420-B; BenQ, Taipei, Taiwan) projecting a $12 \mathrm{~mm}$-wide cross (eye-to-screen distance $63 \mathrm{~cm}$ ). Sufficient the eye velocity vector (PEV) while applying increasing stimulation currents from 0 to $475 \mu \mathrm{A}$. c Average ecVEMP response. $P_{1}$ and $N_{1}$ mark the location of the first positive and second negative peak of the response, respectively, to stimulation currents ranging from 0 to $475 \mu \mathrm{A}$. Note that each of the panels represents a different response. Consequently, the vertical axes of each graph have different scales (see the scale bars in each panel)

SCM tension was obtained by having the patient turn the shoulders slightly. This non-standard patient configuration was necessary to allow simultaneous recording of eye movements and to limit patient fatigue resulting from repeated testing. ecVEMP results for each experimental trial were amplified, averaged, and imported into MATLAB R2018b (The Mathworks Inc., Natick, Massachusetts, USA). The signals were then low-pass filtered at $500 \mathrm{~Hz}$ using a ninthorder IIR filter with zero frequency shift. The latencies and amplitudes of the positive $\left(P_{1}\right)$ and negative $\left(N_{1}\right)$ peaks were determined using the Matlab function "findpeaks". When multiple peaks were identified by this function for a given wave, the optimum peak was selected in consensus by four experienced clinical observers (authors $\mathrm{AB}, \mathrm{NG}, \mathrm{SC}, \mathrm{MR}$ and APF). The latency of the ecVEMPs was calculated as 
the latency of the first peak of muscular contraction $\left(P_{1}\right)$, which corresponds to the initiation of the neck movement.

After each stimulation trial, the patient had to report the self-perceived intensity of the stimulus using the clinical $0-8$ visual-analog scale ( 0 -no perception, 8 - too strong) used for fitting cochlear implant patients in our center. Patients were also asked to describe the percept. Only percepts that could be identified as vestibular in consensus between experimenters were considered (see also [7]). For example, percepts evoking motion or disorientation were included, while percepts evoking sound, pain, or tickling were not considered.

\section{Data analysis and statistics}

All analyses were carried out with SigmaPlot 14 (Systat Software, San Jose, CA, USA) and will be presented in "Results".

\section{Results}

Figure 2 compares the median latencies of the average eVOR and ecVEMP responses for trials with a single stimulating pulse of $200 \mu$ s phase width and maximum current intensity (see also Fig. 3 for individual growth functions). Five electrodes were tested in three participating patients. The fastest response observed was that of the ${ }_{\mathrm{LAT}} \mathrm{eVOR}$ (median latency $8.0 \mathrm{~ms}$ ). The ecVEMPs had a median latency of $10.2 \mathrm{~ms}$. The $N_{1}$ peak of the ecVEMPs appeared at median latency of $16.4 \mathrm{~ms}$, and the $P_{1}$ of the eVOR response at a median latency of $21.0 \mathrm{~ms}$. Individual results and group medians (25th-75th percentiles) are presented in detail in Table 2.

Figure 3 compares the growth functions of the responses of the three vestibular pathways recorded simultaneously and elicited with the single-pulse, $200 \mu$ s phase width stimulus profile. To allow comparison between responses, amplitude values were normalized to the maximum obtained per electrode and per patient, for each response. The dynamics of the growth functions were variable across pathways, across subjects, and even across electrodes within subjects. For S1-SAN and S1-LAN, the growth functions of eVOR and ecVEMP responses were practically identical, increasing monotonically with increasing current. Vestibular percept intensities showed a step-like growth function. The responses appeared at the same stimulation current level (i.e., activation thresholds) for the three pathways. In the case of S2-SAN, all pathways were activated in a similar fashion. However, growth functions and were slightly different for the three pathways for S2-LAN, and the eVOR and ecVEMPs were activated at lower currents than that required to elicit a vestibular percept. For S3-PAN, the eVOR pathway was activated at lower currents than the other responses.

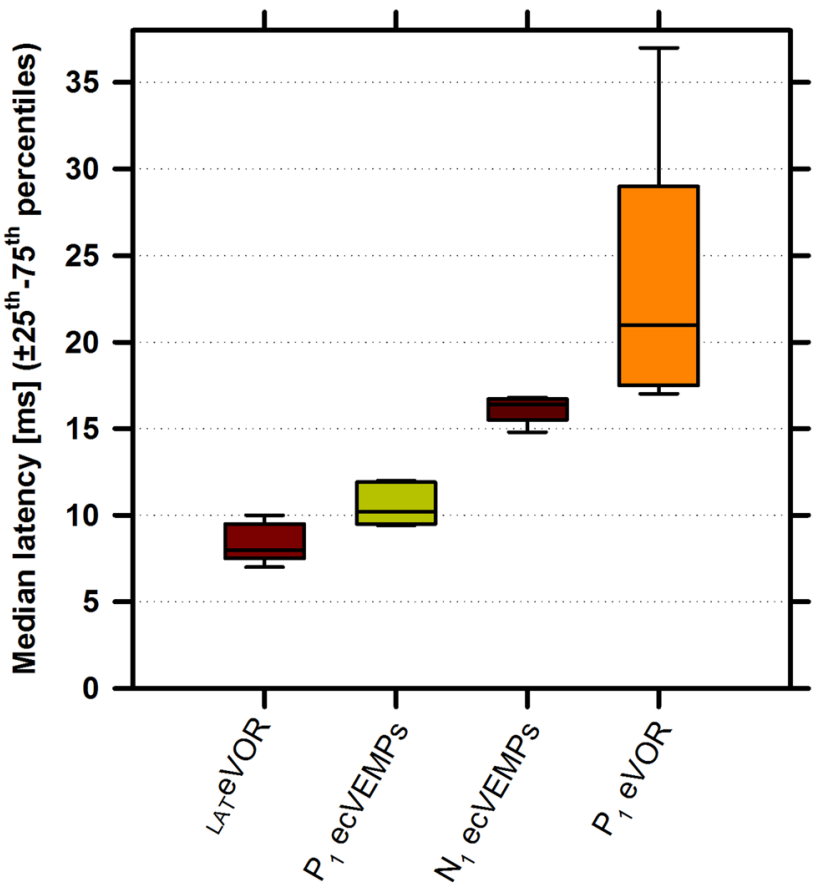

Fig. 2 Latencies of the eVOR and ecVEMP responses elicited upon stimulation with a biphasic, charge-balanced, cathodic-first current pulse of $200 \mu$ s per phase at the UCL (see also Fig. 3). Box plots indicate median values, 25th and 75th percentile values (coloured boxes) as well as 10th and 90th percentile values (error bars) for all subjects and all electrodes tested. Three patients participated in this experiment (S1, S2 and S3), in whom a total of five electrodes were tested (S1-SAN, S1-LAN, S2-SAN, S2-LAN and S3-PAN)

Vestibular percepts showed the highest activation threshold and showed the steepest growth function.

It is interesting to point out that in the experiments presented here, percept intensities remained relatively low (maximum 3), and therefore, UCL levels were always determined by the maximum current amplitudes allowed by the device. In this particular experimental paradigm, patients did not report any non-vestibular sensations (e.g., sound, pain). Vestibular percepts were generally described as a very slight feeling of motion, balancing, or neck stiffness.

The next step of our investigation was to explore the influence of the stimulation profile on all vestibular pathways, measured simultaneously. Only S1 was available for this additional experiment, where stimulation through SAN and LAN electrodes was investigated (Fig. 4). Two phase durations (50 $\mu$ s and $200 \mu \mathrm{s}$ ) and pulse train durations (single pulse and train of four pulses at $1600 \mathrm{pps}$ ) were evaluated. The trend of the results was similar for the SAN (left column) and LAN (right column) electrodes for eVOR and ecVEMP responses. Both reflex responses increased monotonically with increasing current, and a statistically significant correlation was found (Pearson's linear regression analyses; $p<0.001 ; R^{2}>0.81$; slopes shown in Fig. 5). 
Fig. 3 Growth function of the normalized amplitude of the $\mathrm{P}_{1}$ eVOR (red plot), the N-P amplitude for ecVEMPs (orange plot), and individual selfreported percept intensity (green plot) versus current amplitude. Each panel represents responses measured simultaneously in one subject and upon stimulation with one vestibular electrode, for a single-pulse stimulation paradigm, $200 \mu$ s phase width

\section{S1 SAN}

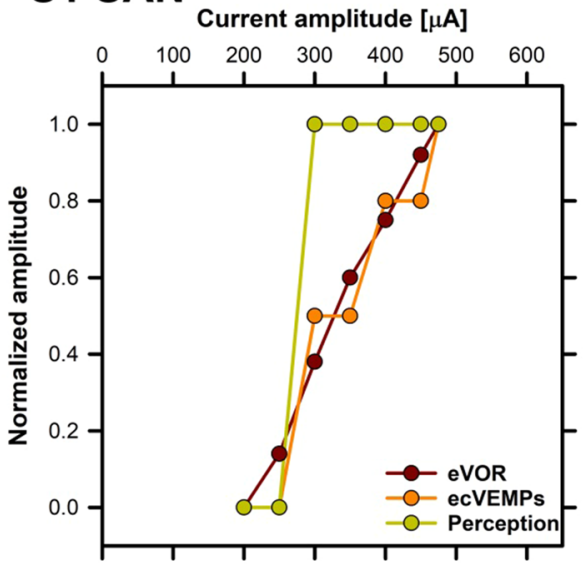

S2 SAN
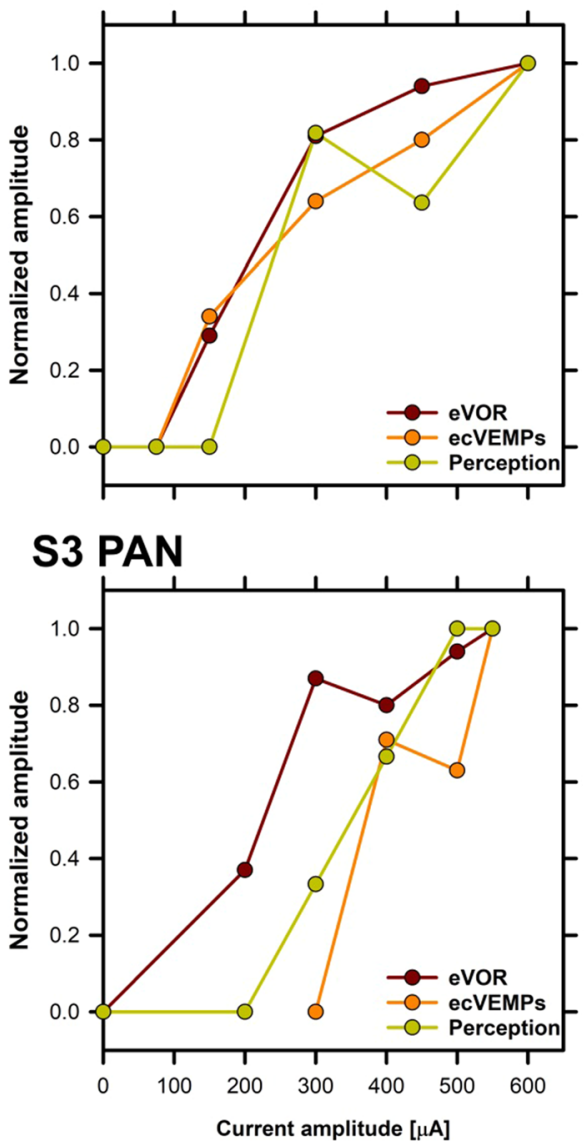

S1 LAN

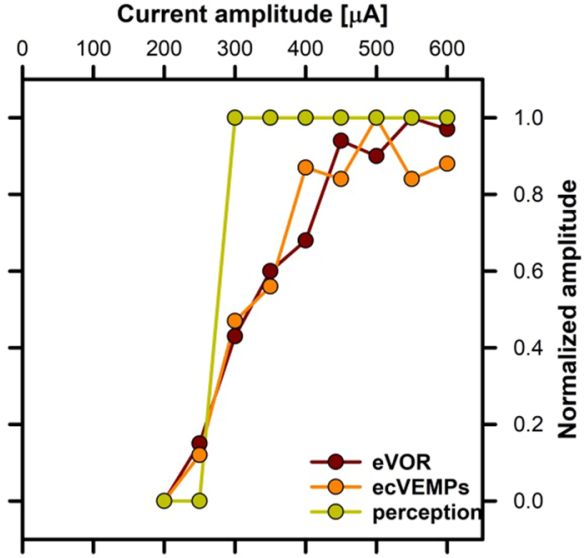

S2 LAN

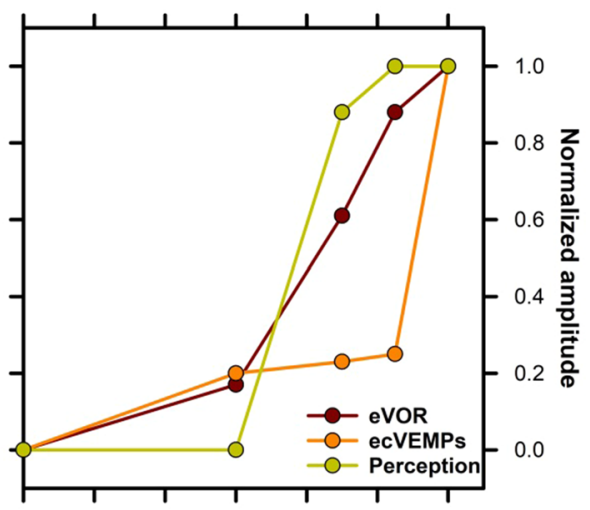

Increasing the phase duration from 50 to $200 \mu$ s (with one pulse) resulted in lower activation thresholds and similar peak amplitudes with around half the stimulation current (i.e., doubling the slope of the growth function; red versus orange bars in Fig. 5). Increasing the number of pulses from 1 to 4 for the $50 \mu$ s pulse width did not decrease activation thresholds but increased the slope of the growth function by nearly a factor of 2 (red versus green bars in Fig. 5). Peak amplitudes were slightly larger with lower currents for the 4-pulse train. Comparing results for the one-pulse profile with $200 \mu$ s pulse width trials to the four-pulse profile with a pulse width of $50 \mu$ s (equal charge per stimulation trial) showed that activation thresholds remained lower for the largest pulse width and that the slopes of the growth functions were $10-40 \%$ steeper for the $200 \mu$ s/phase pulse profile (orange versus green bars in Fig. 5). Maximum peak amplitudes were only slightly larger (0-70\%) with the four-pulse profile, but required double the stimulation current. 
Table 2 Main characteristics of eVOR and cVEMPs elicited upon stimulation with a charge-balanced, cathodic-first, biphasic current pulse of $200 \mu$ s per phase at the maximum intensity (see also Figs. 2 and 3)

\begin{tabular}{|c|c|c|c|c|c|c|c|}
\hline \multicolumn{5}{|l|}{ eVOR } & \multicolumn{3}{|l|}{ ecVEMPs } \\
\hline Subject & Electrode & $\mathrm{LAT}_{\mathrm{eVOR}}[\mathrm{ms}]$ & $P_{1}$ latency $[\mathrm{ms}]$ & $P_{1}$ amplitude $[\% / \mathrm{s}]$ & $P_{1}$ latency $[\mathrm{ms}]$ & $N_{1}$ latency $[\mathrm{ms}]$ & $\begin{array}{l}N-P \\
\text { amplitude } \\
{[\mu \mathrm{V}]}\end{array}$ \\
\hline \multicolumn{8}{|c|}{ Single pulse, $200 \mu \mathrm{s} / \mathrm{phase}$} \\
\hline $\mathrm{S} 1$ & SAN & 7.00 & 21 & 15.36 & 10.20 & 16.60 & 12.25 \\
\hline $\mathrm{S} 1$ & LAN & 8.00 & 21 & 17.16 & 12.00 & 16.40 & 11.60 \\
\hline $\mathrm{S} 2$ & SAN & 8.00 & 18 & 12.87 & 11.80 & 16.20 & 10.07 \\
\hline S2 & LAN & 9.00 & 17 & 4.44 & 9.40 & 14.80 & 5.13 \\
\hline \multirow[t]{4}{*}{ S3 } & PAN & 10.00 & 37 & 4.65 & 9.60 & 16.80 & 7.09 \\
\hline & Median & 8.00 & 21 & 12.87 & 10.20 & 16.40 & 10.07 \\
\hline & 25 th percentile & 8.00 & 18 & 4.65 & 9.60 & 16.20 & 7.09 \\
\hline & 75th percentile & 9.00 & 21 & 15.36 & 11.80 & 16.60 & 11.60 \\
\hline \multicolumn{8}{|c|}{ Single pulse, $50 \mu \mathrm{s} / \mathrm{phase}$} \\
\hline S1 & SAN & 10.00 & 22 & 12.49 & 10.60 & 15.80 & 8.86 \\
\hline \multirow[t]{4}{*}{ S1 } & LAN & 9.00 & 20 & 15.75 & 10.80 & 16.00 & 11.07 \\
\hline & Median & 9.50 & 21 & 14.12 & 10.70 & 15.90 & 9.97 \\
\hline & 25th percentile & 9.25 & 20.5 & 12.49 & 10.65 & 15.85 & 9.41 \\
\hline & 75th percentile & 9.75 & 21.5 & 14.94 & 10.75 & 15.95 & 10.52 \\
\hline \multicolumn{8}{|c|}{ A train of four pulses, $50 \mu \mathrm{s} / \mathrm{phase}, 1600 \mathrm{pps}$} \\
\hline S1 & SAN & 9.00 & 22 & 25.05 & 11.20 & 16.26 & 16.26 \\
\hline \multirow[t]{4}{*}{ S1 } & LAN & 9.00 & 21 & 20.27 & 11.60 & 13.10 & 13.10 \\
\hline & Median & 9.00 & 21.5 & 22.66 & 11.40 & 14.68 & 14.68 \\
\hline & 25 th percentile & 9.00 & 21.25 & 21.47 & 11.30 & 13.89 & 13.89 \\
\hline & 75th percentile & 9.00 & 21.75 & 23.86 & 11.50 & 15.47 & 15.47 \\
\hline
\end{tabular}

Overall, perceived intensities remained very low across trials and stimulation currents. The growth function had a step-like behavior, in contrast to the linear behavior of the other pathways. Evoking vestibular percepts required the lowest currents for the single-pulse trials with $200 \mu$ s pulse width, and the highest stimulation currents for the singlepulse trials with $50 \mu$ s pulse width.

\section{Discussion}

The goal of this study was to explore the simultaneous activation of multiple vestibular pathways. Specifically, we compared their main characteristics (latencies, amplitude growth functions) and explored different stimulation profiles. eVOR, ecVEMPs, and perceptual responses could be evoked in all three tested subjects with five different vestibular electrodes. In this study, reflex pathways showed relatively similar amplitude growth functions that increased monotonically with the amount of charge per stimulus delivered in each case. The amplitude growth function of perceptual responses was different, with perceived intensities remaining quite low and showing a step-like behavior. Finally, the parametric variations attempted here influenced activation thresholds and the growth function of each vestibular pathway. Increasing the phase duration ( $50 \mu$ s vs $200 \mu$ s) doubled the slope of the growth function, even when equal charge was delivered per stimulation trial. This might be expected from nerve stimulation at pulse durations well below the chronaxie value (see also [37]).

Response latencies are due to the specific physical constraints of the neural circuitry underlying the corresponding pathways (i.e., synaptic neural delays, neural conduction delays, muscle activation times, and pathway length) [39]. Here we present, for the first time in humans, the compared average latencies of the electrically evoked vestibular reflex pathways. Our results are in accordance with the latencies reported both in human and animal studies for "natural" and electrical stimulation [39-42]. Note that in this study we compared the latency of the initiation of the eVOR ( $\mathrm{LAT}^{\mathrm{e}}$ VOR) with the latency of $P_{1}$ of the ecVEMPs, because both correspond to the actual initiation of the eye/neck movement. However, in the future, it might be interesting to explore the latencies of the onset of muscular contraction of cervical and ocular myogenic potentials, which could be more informative on the actual time that the signal takes to 


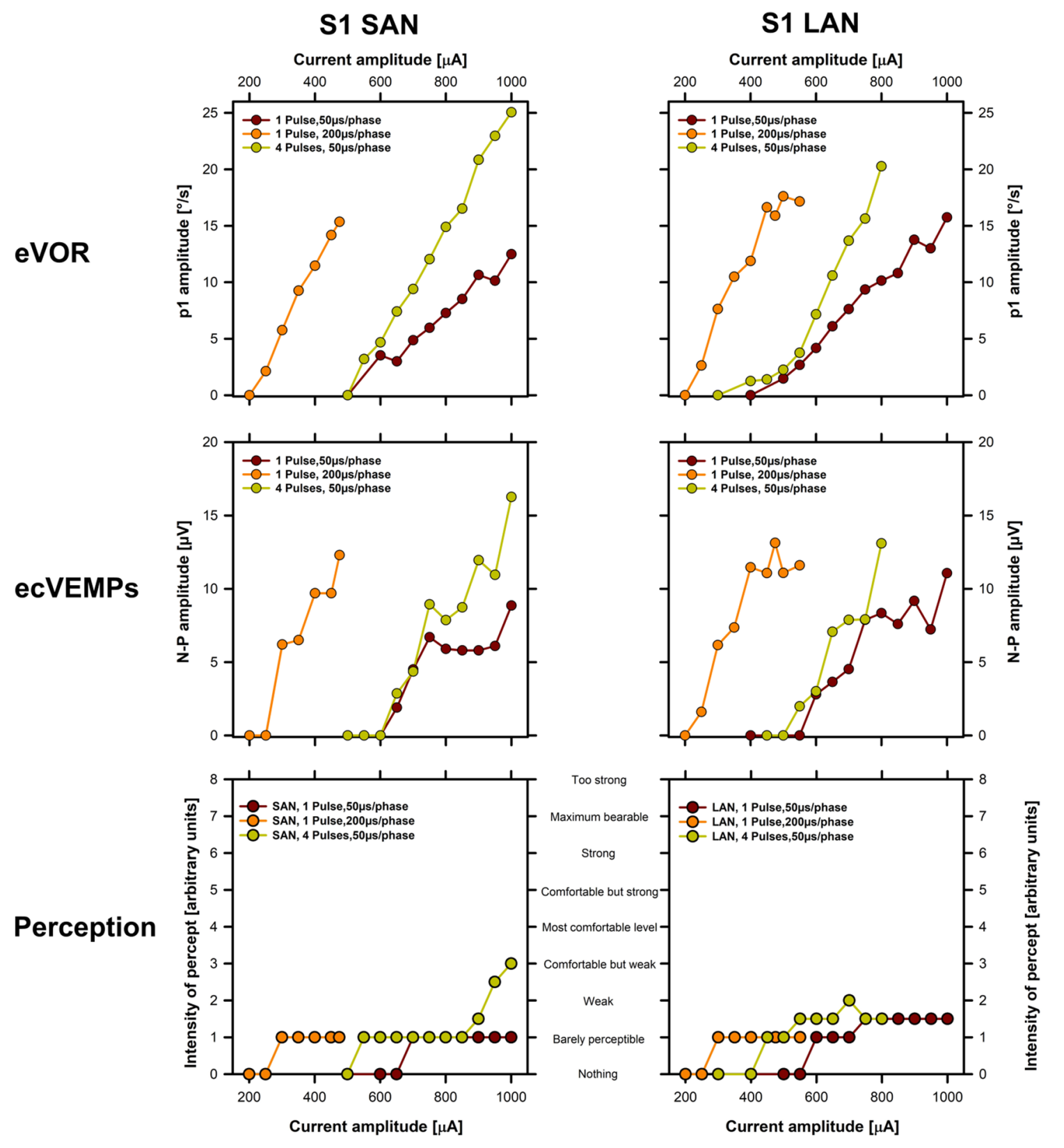

Fig. 4 Growth function of the amplitude of the eVOR P1, the ecVEMPs P1 and individual self-reported percept recorded using different stimulation profiles (single pulse, $50 \mu$ s per phase-red plot; single pulse, $200 \mu$ s per phase-orange plot; train of four pulses,

reach the muscle, providing actual information about the neural pathway without considering the characteristics of the muscle itself.

Comparing the growth functions of the vestibular reflexes shows that, in some cases, the dynamics of the different pathways were not always identical across subjects and that activation thresholds might differ between pathways (Fig. 3). This inter-subject variability may be due to the relative spread of current from ampulla to otolith organs
$50 \mu$ s per phase, 1600 pps-green plot) versus current amplitude. Only subject S1 was available for this experiment where two electrodes were investigated (electrode SAN-left column and LANright column)

being different in each case. Moreover, the patient profiles were different, which could also explain the differences. For example, while patients S1 and S2 suffered from severe acute bilateral vestibular loss caused by a trauma $\mathrm{S} 3 \mathrm{had}$ a long duration, congenital bilateral vestibular loss. Furthermore, in S3, the active electrode was the PAN electrode (stimulated at an extralabyrinthine location), while in $\mathrm{S} 1$ and $\mathrm{S} 2$, the stimuli were delivered through the SAN and LAN electrodes (in an intralabyrinthine configuration). However, 
S1 SAN
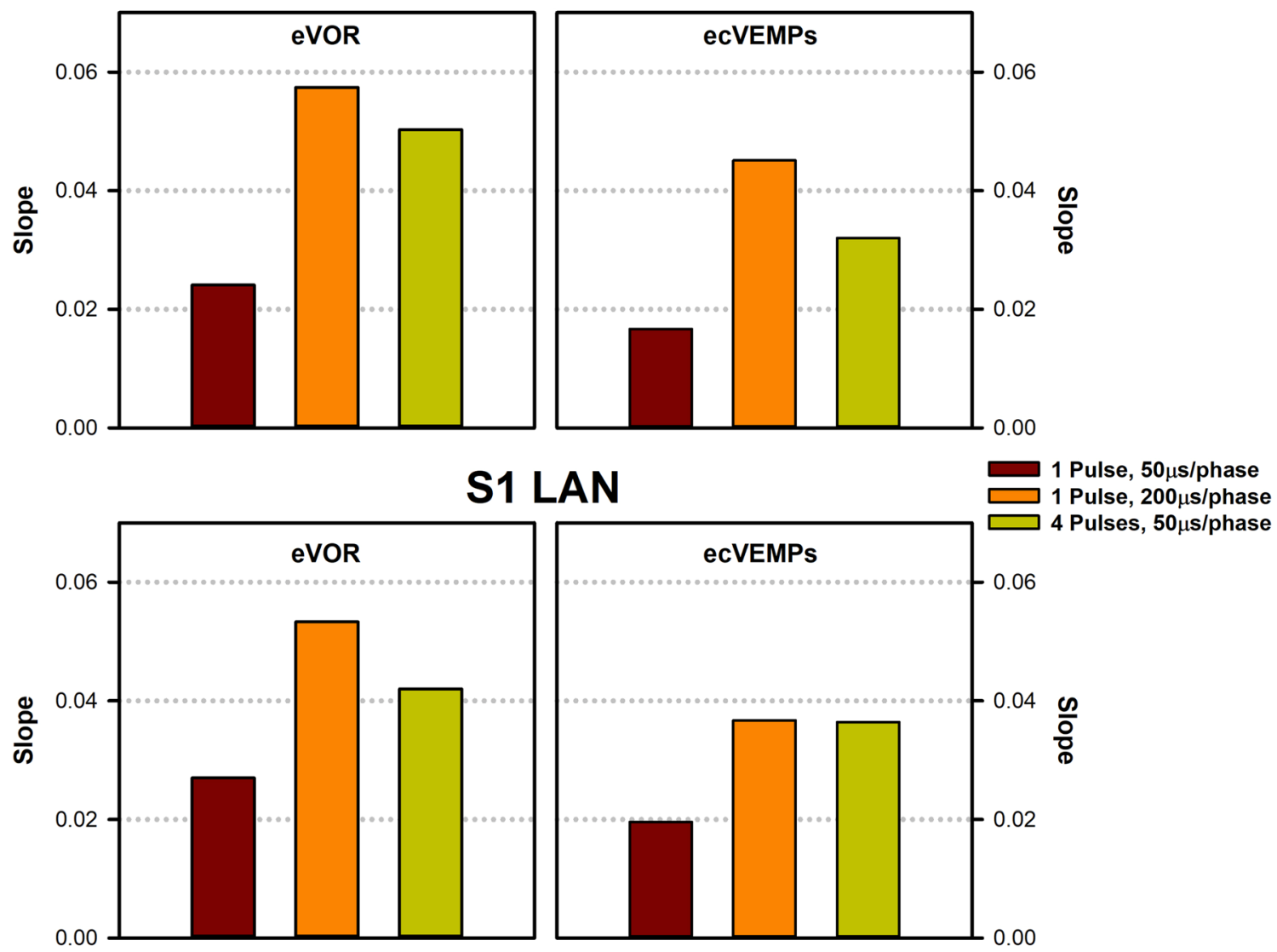

Fig. 5 Slope of the growth function of eVOR (left panels) and ecVEMP responses, calculated using linear regression analyses of the normalized data versus stimulation current (see also Fig. 4). Three stimulation paradigms are compared: single pulse, $50 \mu$ s per phasered plot; single pulse, $200 \mu$ s per phase-orange plot; train of four pulses, $50 \mu$ s per phase, 1600 pps-green plot) versus current ampli-

the small sample size in this study makes it difficult to draw any definite conclusions with respect to the relationship of the location of the active electrodes and the responses.

eVOR and ecVEMP responses increased monotonically with increasing current while perceptual responses showed a step-like growth function (Fig. 4). On the one hand, this observation could be explained because we stimulated with a non-physiological electrical stimulus in isolation (i.e., in the absence of any convergent extra-vestibular stimuli which naturally contribute to perception). This can potentially limit the grow function of intensity perception. On the other hand, this observation could be also explained by the existence of different kinds of vestibular afferent fibers in each vestibular pathway. Morphological and physiological properties of the vestibular afferents (e.g., axon diameter, discharge regularity) allow them to be classified as either regular or irregular [43, 44]. For instance, irregular afferents tend to have thicker axons tude. Only subject S1 was available for this experiment where two electrodes were investigated (electrode SAN-upper line and LANlower line). Note that the slope of the growth function for the intensity of percepts was not calculated since it showed a step-like behavior (not a monotonical increase with respect to stimulation current)

[45] and thresholds 5-10 times lower than regular afferents [46]. Despite overlap of regular and irregular afferents in each of the subdivisions of the vestibular nuclei [47], electrophysiological studies suggest that the different pathways might be primarily mediated by one type of afferent, with a higher proportion of irregular afferents in the VTC pathway [46]. While these results are still under discussion, these differences in afferent populations combined with the complexity of neuronal connections of the VTC pathway could at least partly explain the observed differences. Moreover, due to the very brief stimulation trials (i.e., the use of 1-4 pulses), the self-perceived stimulation intensities from all patients remained very low, which limited proper evaluation of the growth function. In previous studies [37], where different stimulation profiles and longer stimuli were used, we observed much higher percept intensities. We will attempt to investigate the VTC pathway in more detail in subsequent studies using 
alternative objective methods (e.g., imaging, electrophysiological) and adapted stimulation profiles.

Finally, to explore the effects of different stimulation paradigms on vestibular responses, three different stimulation profiles were tested on one patient (S1). This investigation revealed that increasing the electrical charge in the stimulus ( $200 \mu$ s vs $50 \mu$ s per phase) enhanced all responses (Fig. 4). In addition, while the growth functions of eVOR and ecVEMP responses to two different paradigms containing the same electrical charge (single pulse at $200 \mu \mathrm{s} /$ phase vs train of four-pulse at $50 \mu \mathrm{s} /$ phase, $1600 \mathrm{pps}$ ) were similar, the slopes were steeper for the single pulse at $200 \mu \mathrm{s} /$ phase. In other words, a single $200 \mu$ s pulse required only half of the current required to evoke an equivalent response with four $50 \mu$ s pulses presented at 1600 pps. Therefore, the $200 \mu$ s pulse width seems to be a good balance between efficient activation of the vestibular pathways and lower energy consumption. This difference can be related to the physiological features of the nerve. The absolute refractory period of a nerve varies between 0.5 and $1 \mathrm{~ms}$ and corresponds to the recovery time of the membrane (during which it is impossible to generate additional action potentials). This period is followed by a relative refractory period of approximately $10 \mathrm{~ms}$ during which the threshold for eliciting spikes is increased. Stimulating with four pulses at $1600 \mathrm{pps}$ means one pulse is applied every $0.6 \mathrm{~ms}$. Consequently, after the first short stimulation pulse, the next might be taking place during the relative refractory period of the nerve which could at least partially explain the observed differences [48].

\section{Limitations and future work}

The main limitation of this study is the small number of patients included, especially for the investigation of different stimulation profiles for which only S1 was available. Indeed, at the present time, only a few patients having received our prototype vestibular implant device are available for testing. This is a common limitation for all current vestibular implant studies (see, e.g., [17, 27, 32]). Furthermore, these experiments are long and time consuming (approximately four hours per patient). We hope that as more patients and more groups get involved in this research, these results will be reproduced in larger cohorts to further validate the observed trends and effects. Note, however, that all subjects showed similar trends. Therefore, despite large intersubject variability, important systematic features could be described.

Overall, the trend between the eVOR and ecVEMPs across different paradigms was similar (Fig. 5). However, the slopes of the eVOR responses seem to be steeper than that of ecVEMPS. While this difference might be related to the strength of activation of each pathway, other parameters inherent to the experiment (e.g.; SCM muscle tension during ecVEMPs recordings) might also have influenced this result.
Another limitation of this study was the evaluation of the VTC pathway through subjective reports of patients and upon very short stimulation trials. Research is currently underway to extend the exploration of these pathways with the use of objective electrophysiological measures.

In summary, this study opens the door to the possibility of selectively activating one pathway or the other, at least in some cases. However, this issue clearly deserves further and more detailed investigation to determine the actual possibilities for selective stimulation, the best strategies, and the functional significance of the contribution of each pathway to the overall rehabilitation process.

Acknowledgements Open access funding provided by University of Geneva. The authors thank the patients for their time and patience, and Carolyn Garnham for critical review of the manuscript.

Funding This study was carried out with the financial support of a private foundation AURIS, www.fondationauris.org.

\section{Compliance with ethical standards}

Conflicts of interest $\mathrm{AB}, \mathrm{CC}, \mathrm{NG}, \mathrm{RVdB}$ and $\mathrm{APF}$ have received travel and research grants from MED-EL Elektromedizinische Geräte $\mathrm{GmbH}$ (Innsbruck, Austria).

Ethical standards This protocol was approved and carried out in accordance with the recommendations of the local ethics committee (Geneva University Hospitals NAC 11-080) and was designed in accordance with the declaration of Helsinki for which patients provided written informed consent.

Open Access This article is licensed under a Creative Commons Attribution 4.0 International License, which permits use, sharing, adaptation, distribution and reproduction in any medium or format, as long as you give appropriate credit to the original author(s) and the source, provide a link to the Creative Commons licence, and indicate if changes were made. The images or other third party material in this article are included in the article's Creative Commons licence, unless indicated otherwise in a credit line to the material. If material is not included in the article's Creative Commons licence and your intended use is not permitted by statutory regulation or exceeds the permitted use, you will need to obtain permission directly from the copyright holder. To view a copy of this licence, visit http://creativecommons.org/licenses/by/4.0/.

\section{References}

1. Ward BK, Agrawal Y, Hoffman HJ, Carey JP (2013) Prevalence and impact of Bilateral Vestibular Deficiency (BVD): results from the 2008 United States National Health Interview Survey. JAMA Otolaryngol Head Neck Surg 139(August 1):803-810

2. Guinand N, Boselie F, Guyot JP, Kingma H (2012) Quality of life of patients with bilateral vestibulopathy. Ann Otol Rhinol Laryngol 121(7):471-477

3. Krebs DE, Gill-Body KM, Parker SW, Ramirez JV, WernickRobinson M (2003) Vestibular rehabilitation: useful but not universally so. Otolaryngol Head Neck Surg 128(2):240-250 
4. Zingler VC et al (2008) Follow-up of vestibular function in bilateral vestibulopathy. J Neurol Neurosurg Psychiatry 79(3):284-288

5. Guyot JP, Perez Fornos A (2019) Milestones in the development of a vestibular implant. Curr Opin Neurol 32(1):145-153

6. Merfeld DM, Lewis RF (2012) Replacing semicircular canal function with a vestibular implant. Curr Opin Otolaryngol Head Neck Surg 20(5):386-392

7. Guinand $N$ et al (2015) Vestibular implants: 8 years of experience with electrical stimulation of the vestibular nerve in 11 patients with bilateral vestibular loss. Orl 77:227-240

8. Cohen B, Suzuki J-I (1963) Eye movements induced by ampullary nerve stimulation' difficult because of its position in the temporal. Am J Physiol 204(2):347-351

9. Suzuki JI, Cohen B (1964) Head, eye, body and limb movements from semicircular canal nerves. Exp Neurol 10(5):393-405

10. Sierra H, Cordova M, Chen CSJ, Rajadhyaksha M (2015) Confocal imaging-guided laser ablation of basal cell carcinomas: an ex vivo study. J Invest Dermatol 135(2):612-615

11. Davidovics NS, Fridman GY, Della Santina CC (2012) Comodulation of stimulus rate and current from elevated baselines expands head motion encoding range of the vestibular prosthesis. Exp Brain Res 218(3):389-400

12. Dai $C$ et al (2013) Directional plasticity rapidly improves 3D vestibulo-ocular reflex alignment in monkeys using a multichannel vestibular prosthesis. JARO J Assoc Res Otolaryngol 14(6):863-877

13. Davidovics NS, Rahman MA, Dai C, Ahn J, Fridman GY, Della Santina CC (2013) Multichannel vestibular prosthesis employing modulation of pulse rate and current with alignment precompensation elicits improved vor performance in monkeys. JARO J Assoc Res Otolaryngol 14(2):233-248

14. Lewis RF, Haburcakova C, Gong W, Makary C, Merfeld DM (2010) Vestibuloocular reflex adaptation investigated with chronic motion-modulated electrical stimulation of semicircular canal afferents. J Neurophysiol 103(2):1066-1079

15. Mitchell DE, Della Santina CC, Cullen KE (2016) Plasticity within non-cerebellar pathways rapidly shapes motor performance in vivo. Nat Commun 7(May):1-13

16. Mitchell DE, Dai C, Rahman MA, Ahn JH, Della Santina CC, Cullen KE (2013) Head movements evoked in alert rhesus monkey by vestibular prosthesis stimulation: implications for postural and gaze stabilization. PLoS ONE 8(10):1-12

17. Macias AR, De Miguel AR, Montesdeoca IR, Barreiro SB, González JCF (2020) Chronic electrical stimulation of the otolith organ: preliminary results in humans with bilateral vestibulopathy and sensorineural hearing loss. Audiol Neurotol 25(1-2):79-90

18. Hageman $\mathrm{KN}$ et al (2020) Binocular 3D otolith-ocular reflexes: responses of chinchillas to prosthetic electrical stimulation targeting the utricle and saccule. J Neurophysiol 123(1):259-276

19. Della Santina CC, Migliaccio AA, Patel AH (2007) A multi-channel semicircular canal neural prosthesis using electrical stimulation to restore 3D vestibular sensation. IEEE Trans Biomed Eng 54(6):1016-1030

20. van de Berg R, Guinand N, Guyot JP, Kingma H, Stokroos RJ (2012) The modified ampullar approach for vestibular implant surgery: Feasibility and its first application in a human with a long-term vestibular loss. Front Neurol FEB(February):1-7

21. van de Berg R et al (2017) The vestibular implant: hearing preservation during intralabyrinthine electrode insertion-a case report. Front Neurol 8(APR):1-7

22. Stultiens JJA, Postma AA, Guinand N, Pérez Fornos A, Kingma $\mathrm{H}$, van de Berg R (2020) Vestibular implantation and the feasibility of fluoroscopy-guided electrode insertion. Otolaryngol Clin North Am 53(1):115-126

23. Kos MI, Feigl G, Anderhuber F, Wall C, Fasel JHD, Guyot JP (2006) Transcanal approach to the singular nerve. Otol Neurotol 27(4):542-546
24. Fornos AP et al (2014) Artificial balance: restoration of the vestibulo-ocular reflex in humans with a prototype vestibular neuroprosthesis. Front Neurol 5APR(April):1-11

25. Fornos AP et al (2019) Cervical myogenic potentials and controlled postural responses elicited by a prototype vestibular implant. J Neurol 266(1):33-41

26. Guinand $\mathrm{N}$ et al (2017) The video head impulse test to assess the efficacy of vestibular implants in humans. Front Neurol 8:600

27. Boutros PJ et al (2019) Continuous vestibular implant stimulation partially restores eye-stabilizing reflexes. JCI Insight 4(22):1-19

28. van de Berg R et al (2015) The vestibular implant: frequencydependency of the electrically evoked vestibulo-ocular reflex in humans. Front Syst Neurosci 8(JAN):1-12

29. Guinand $\mathrm{N}$ et al (2016) Restoring visual acuity in dynamic conditions with a vestibular implant. Front Neurosci 10(DEC):1-6

30. Phillips $C$ et al (2013) Postural responses to electrical stimulation of the vestibular end organs in human subjects. Exp Brain Res 229(2):181-195

31. Fornos AP et al (2019) Cervical myogenic potentials and controlled postural responses elicited by a prototype vestibular implant. J Neurol 266(s1):33-41

32. Rubinstein JT, Ling L, Nowack A, Nie K, Phillips JO (2020) Results from a second-generation vestibular implant in human subjects. Otol Neurotol 41(1):68-77

33. Straka H, Dieringer N (2004) Basic organization principles of the VOR: lessons from frogs. Prog Neurobiol 73(4):259-309

34. Cullen KE (2016) Physiology of central pathways, 1st edn. In: Handbook of Clinical Neurology, vol 137, Elsevier B.V.

35. Goldberg J, Peterson BW (1986) Reflex and mechanical contributions to head stabilization in alert cats. J Neurophysiol 56(3):857-875

36. Ezure K, Sasaki S (1978) Frequency-response analysis of vestibular-induced neck reflex in cat. I. Characteristics of neural transmission from horizontal semicircular canal to neck motoneurons. J Neurophysiol 41(2):445-458

37. Crétallaz C et al (2020) Influence of systematic variations of the stimulation profile on responses evoked with a vestibular implant prototype in humans. J Neural Eng 17:1-14

38. Feigl GC et al (2009) Superior vestibular neurectomy: a novel transmeatal approach for a denervation of the superior and lateral semicircular canals. Otol Neurotol 30(5):586-591

39. Huterer M, Cullen KE (2002) Vestibuloocular reflex dynamics during high-frequency and high-acceleration rotations of the head on body in Rhesus monkey. J Neurophysiol 88(1):13-28

40. Collewijn H, Smeets JBJ (2000) Early components of the human vestibulo-ocular response to head rotation: latency and gain. J Neurophysiol 84(1):376-389

41. Watson SRD, Colebatch JG (1998) Vestibular-evoked electromyographic responses in soleus: a comparison between click and galvanic stimulation. Exp Brain Res 119(4):504-510

42. Watson SRD, Colebatch JG (1998) Vestibulocollic reflexes evoked by short-duration galvanic stimulation in man. J Physiol 513(2):587-597

43. Baird RA, Desmadryl G, Fernandez C (1988) The vestibular nerve of the chinchilla. II. Relation between afferent response properties and peripheral innervation patterns in the semicircular canals. $\mathrm{J}$ Neurophysiol 60(1):182-203

44. Goldberg JM (2000) Afferent diversity and the organization of central vestibular pathways. Exp Brain Res 130(3):277-297

45. Goldberg JM, Lysakowski A, Fernández C (1990) Morphophysiological and ultrastructural studies in the mammalian cristae ampullares. 49:89-102

46. Boyle R, Goldberg JM, Highstein SM (1992) Inputs from regularly and irregularly discharging vestibular nerve afferents to secondary neurons in squirrel monkey vestibular nuclei. III. Correlation 
with vestibulospinal and vestibuloocular output pathways. J Neurophysiol 68(2):471-484

47. Sato F, Sasaki H, Ishizuka N, Sasaki S-I, Mannen H (1989) Morphology of single primary vestibular afferents originating from the horizontal semicircular canal in the cat. J Comp Neurobiol
290(3):423-439. https://onlinelibrary.wiley.com/toc/10969 $861 / 1989 / 290 / 3$

48. De Fabrizio Gabbiani SJC (2010) Mathematics for neuroscientists, 2nd edn. REFRACTORY PERIOD, Academic Press, p 15.2 